\title{
Excess low energy photon pairs from pion annihilation at the chiral phase transition
}

\author{
M.K. Volkov, ${ }^{\mathrm{a}, 1}$ E.A. Kuraev, ${ }^{\mathrm{a}, 2}$ D. Blaschke, ${ }^{\mathrm{b}}$ G. Röpke, ${ }^{\mathrm{a}, \mathrm{b}}$ \\ S. Schmidt $b, c, 3$ \\ ${ }^{a}$ Bogoliubov Laboratory for Theoretical Physics \\ Joint Institute for Nuclear Research, \\ 141980 Dubna, Russia \\ ${ }^{\mathrm{b}}$ Fachbereich Physik, Universität Rostock \\ D-18051 Rostock, Germany \\ ${ }^{\mathrm{c}}$ School of Physics and Astronomy \\ Raymond and Beverly Sackler Faculty of Exact Sciences \\ Tel Aviv University, $69978 \mathrm{Tel}$ Aviv, Israel
}

\begin{abstract}
The photon pair production by pion annihilation in a hot and dense medium at the chiral phase transition is investigated within a chiral quark model. As a direct consequence of this transition the $\sigma$ meson appears as a bound state in the domain of temperatures and chemical potentials where the condition $M_{\sigma}(T, \mu) \approx 2 M_{\pi}(T, \mu)$ is fulfilled. This effect results in a strong enhancement of the cross section for the pion annihilation process $2 \pi \rightarrow 2 \gamma$ compared with the vacuum case. The calculation of the photon pair production rate as function of the invariant mass shows a strong enhancement and narrowing of the $\sigma$ meson resonance at threshold due to chiral symmetry restoration.
\end{abstract}

PACS number(s): 05.70.Jk, 11.30.Rd, 13.40.-f, 14.40.-n, 25.75.-q

Key words: two-photon spectra, chiral symmetry, sigma meson, pion annihilation

1 Supported by INTAS grant No. W 94-2915

2 Supported by INTAS grant No. W 93-0239

3 Supported by MINERVA Foundation

Preprint submitted to Elsevier Preprint

3 June 2021 


\section{Introduction}

The investigation of hadron properties at finite temperature and density is very important in order to provide insight into phenomena occuring in the vicinity of the transition to the hypothetical quark-gluon plasma state of matter which is characterized by hadron deconfinement and restoration of chiral symmetry. These studies become particularly interesting since in ultra-relativistic heavy ion collision experiments performed and planned at BNL Brookhaven and CERN Geneva one is able to create matter at the extreme densities and temperatures necessary for the phase transition. Different effects which were suggested as possible signatures for the chiral/deconfinement transition such as $J / \Psi$ suppression, strangeness enhancement and low-mass dilepton enhancement have been observed. For a recent discussion of these experimental findings, see [1]. The most direct probes from the suspected quark-gluon plasma state and the subsequent hadronisation transition are photons and dileptons since they leave the hot and dense matter at an early stage without suffering strong interactions.

The most abundant secondaries produced in heavy ion collisions are pions which evolve through a hot and dense hadron gas state until the freeze-out. Therefore it is interesting to investigate the behaviour of such a gas at large temperature and/or density, in particular to study its radiation. The main channel of this radiation is the annihilation of two pions into a virtual photon which decays into a lepton pair. With help of this reaction it is possible to study the behaviour of the intermediate $\rho$ meson under the influence of a hot and dense medium $[2-4]$.

Besides of this dominant radiation process of the pion gas, the annihilation of two pions into two photons is of particular importance since its cross section is sensitive to changes in the $\sigma$ meson properties which occur in the vicinity of the chiral restoration transition. In the vacuum this reaction contributes to the amplitude with noticeable probability only via the Born diagrams for the charged pions. Compared with the vacuum case, with increasing temperatures and densities the $\sigma$ meson [5-8] changes its character [9] from a broad resonance with a large decay width into the two-pion channel to a bound state below the two-pion threshold $M_{\sigma}(T, \mu) \leq 2 M_{\pi}(T, \mu)$ and its contribution to the cross section of the process $2 \pi \rightarrow 2 \gamma$ overwhelms that of the Born terms [10]. This effect can be understood as an inevitable consequence of the transition from a phase of matter with restored chiral symmetry (i.e. degenerate scalar and pseudoscalar fields) to a phase where this symmetry is broken and the scalar meson aquires a mass above the two-pion threshold with a large decay width into the two-pion channel. A similar effect has been discussed by Weldon [12] for the soft lepton pair production by pion annihilation in a hot, dense pion gas where due to chiral symmetry restoration the $\sigma$ resonance can give a sizeable contribution to lepton pair spectra provided a charge asymmetry of the pionic medium.

It is the aim of the present paper to investigate the enhancement of low-energy photon pairs from pion annihilation at the chiral/hadronisation transition and to give numerical estimates for both the magnitude of the cross section enhancement (critical scattering) and the photon 
production rate on the basis of a chiral quark model. This effect of a threshold enhancement in the photon pair spectra could indicate chiral symmetry restoration from ultrarelativistic heavy-ion collisions.

The decay of the pion into two quarks appears at higher values of $T$ and $\mu$ and does not play any rôle in the chosen scenario. But it is important to note that in this domain the $\sigma$ meson enters the continuum of $q \bar{q}$ states. The appearence of such a $\sigma$ resonance and the photon pair spectrum in a quark plasma has been studied in [11] and complements the present investigations.

\section{The process $\pi \pi \rightarrow \gamma \gamma$ at finite temperature and chemical potential}

The process $\pi \pi \rightarrow \gamma \gamma$ is described by the Born terms (for charged pions, see Fig. 1) and the quark-substructure terms (for charged and neutral pions, see Fig. 2) t. The box diagrams and the $\sigma$ pole diagram (Fig. 2 b, c) are the most important contributions. Processes with vector mesons $(\rho, \omega)$ in the t-channel are also present but give a negligible contribution.

The Lagrangian describing the diagrams of Fig. 1 has the form

$$
\mathcal{L}=i e A_{\mu}\left(\pi^{-} \partial_{\mu} \pi^{+}-\pi^{+} \partial_{\mu} \pi^{-}\right)+e^{2} \mathcal{A}_{\mu}^{2} \pi^{+} \pi^{-} .
$$

The diagrams of Fig. 2 describe the most important contributions to the $\pi \pi \rightarrow \gamma \gamma$ process in terms of a low energy quark model of QCD in $1 / N_{c}$ approximation. According to this level of description the meson-meson and meson-photon vertices are given by quark-loop diagrams (triangle and box diagrams) which have previously been analysed, e.g., within the Nambu-Jona-Lasinio (NJL) model $[7,8,13]$. In local approximation and for low-energy photons $\left(k^{2}\right.$ approximation) the relevant vertices are given by the following Langrangians

$$
\begin{aligned}
\mathcal{L}_{\text {box }} & =\frac{\alpha}{18 \pi f_{\pi}^{2}}\left(\pi^{+} \pi^{-}+5 \pi^{0} \pi^{0}\right) F_{\mu \nu}^{2}, \\
\mathcal{L}_{\sigma \pi \pi} & =2 m g_{\pi} \sigma\left(2 \pi^{+} \pi^{-}+\pi^{0} \pi^{0}\right), \\
\mathcal{L}_{\sigma \gamma \gamma} & =\frac{5 \alpha}{9 \pi f_{\pi}} \sigma F_{\mu \nu}^{2},
\end{aligned}
$$

where $\alpha=e^{2} / 4 \pi=1 / 137, F_{\mu \nu}=\partial_{\mu} A_{\nu}-\partial_{\nu} A_{\mu}$. The components of the isovector pseudoscalar meson field are denoted by $\pi^{+}, \pi^{-}, \pi^{0} ; \sigma$ is the isoscalar, scalar meson field. The values of the dynamical quark mass $m$, the pion decay constant $f_{\pi}$, the strong pion coupling constant $g_{\pi}=m / f_{\pi}$ as well as the meson masses $M_{\pi}$ and $M_{\sigma}$ at finite temperatures $T$ and chemical potentials $\mu$ are taken from Ref. [14,15] for the case of the NJL model [16].

$\overline{4}$ The Fig. 1 is the first (local) approximation for the diagrams of Fig. 2 a,b 
The amplitude of the process $\pi^{+} \pi^{-} \rightarrow 2 \gamma$ describing the sum of the Born diagrams as well as the $\sigma$ pole and the box graphs is given by

$$
T_{\pi^{+} \pi^{-} \rightarrow \gamma \gamma}=e^{2}\left[2\left(g^{\mu \nu}-\frac{q_{1}^{\mu} q_{2}^{\nu}}{q_{1} k_{1}}-\frac{q_{2}^{\mu} q_{1}^{\nu}}{q_{2} k_{1}}\right)+\mathcal{A}_{\pi^{+} \pi^{-} \rightarrow \gamma \gamma}\left(g_{\mu \nu} k_{1} k_{2}-k_{1}^{\nu} k_{2}^{\mu}\right)\right] e_{\mu}\left(k_{1}\right) e_{\nu}\left(k_{2}\right),
$$

where $q_{i}$ and $k_{i}$ are the momenta of pions and photons, respectively, $e_{\mu}\left(k_{1}\right)$ and $e_{\nu}\left(k_{2}\right)$ denote the polarization vectors of the photons. The amplitude

$$
\mathcal{A}_{\pi^{+} \pi^{-} \rightarrow \gamma \gamma}=\frac{1}{\left(6 \pi f_{\pi}(T, \mu)\right)^{2}}\left[\frac{40 m^{2}(T, \mu)}{M_{\sigma}^{2}(T, \mu)-s-i M_{\sigma}(T, \mu) \Gamma_{\sigma}(T, \mu)} f_{1}(\mu, T)-f_{2}(\mu, T)\right]
$$

includes the quark substructure contributions of the $\sigma$ pole and the box diagrams of Fig. 2 . We refer the reader to Refs. [7,8,13] for details of the derivation of Eqs. (5), (6). Mass and decay width of the $\sigma$ meson are given within this model as

$$
\begin{aligned}
& M_{\sigma}^{2}(T, \mu)=M_{\pi}^{2}(T, \mu)+4 m^{2}(T, \mu) \\
& \Gamma_{\sigma}(T, \mu)=\frac{3\left[M_{\sigma}^{2}(T, \mu)-M_{\pi}^{2}(T, \mu)\right]^{2}}{32 \pi M_{\sigma}(T, \mu) f_{\pi}^{2}(T, \mu)} \sqrt{1-\frac{4 M_{\pi}^{2}(T, \mu)}{M_{\sigma}^{2}(T, \mu)}} .
\end{aligned}
$$

The mass of the $\sigma$ meson is determined by its internal quark substructure and calculated by solving the Bethe-Salpeter equation at finite temperature $T$ and chemical potential $\mu$ in the scalar quark-antiquark channel. In the NJL model this leads unambiguously to relation (7). The width of the $\sigma$ meson (8) is given by the strong decay channel into two pions which is evaluated in the standard way, e.g. within the linear sigma-model, see e.g. [9,12]. The T, $\mu$ dependences of $M_{\sigma}, M_{\pi}$ and $f_{\pi}$ are obtained from the NJL model. The functions $f_{1}(T, \mu)$ and $f_{2}(T, \mu)$ describe the dependence of the quark triangle and the quark box diagrams on temperature and chemical potential $[13,17]$ and are given by

$$
\begin{aligned}
& f_{1}(T, \mu)=1-\frac{3}{2} m^{2}(T, \mu) \int_{0}^{\infty} d k \frac{k^{3}}{E^{6}(k)} \ln \left[\frac{E(k)+k}{E(k)-k}\right][n(k ; T, \mu)+\bar{n}(k ; T, \mu)], \\
& f_{2}(T, \mu)=3 m^{2}(T, \mu) \int_{0}^{\infty} d k \frac{k^{2}}{E^{5}(k)}[1-n(k ; T, \mu)-\bar{n}(k ; T, \mu)] .
\end{aligned}
$$

The Fermi distribution functions $n(k ; \mu, T)=\{1+\exp [(E(k)-\mu) / T]\}^{-1}$ for particles and $\bar{n}(k ; \mu, T)=\{1+\exp [(E(k)+\mu) / T]\}^{-1}$ for antiparticles describe the dependence on temperature and chemical potential for quarks and antiquarks of the energy $E(k)=\sqrt{k^{2}+m^{2}(T, \mu)}$. Note that the occurence of the functions $f_{1,2}(T, \mu)$ is an important point of our study: the local vertices (Fig. 1) of the Born approximation are replaced by quark loop diagrams (Fig. 
2 a,b). Therefore the vertices become non-local and suffer an explicit medium dependence due to the quark substructure.

In the following we use a short notation, where we drop the $T$ - and $\mu$ dependences in the arguments of the medium dependent quantities. For the square of the transition matrix element (5) we obtain

$$
\begin{aligned}
\left|T_{\pi^{+} \pi^{-} \rightarrow \gamma \gamma}(s)\right|^{2} & =4 e^{4}\left(2+M_{\pi}^{4}\left[\frac{1}{\left(k_{1} q_{1}\right)^{2}}+\frac{1}{\left(k_{2} q_{1}\right)^{2}}\right]+2 \frac{\left(q_{1} q_{2}\right)^{2}}{\left(k_{1} q_{1}\right)\left(k_{2} q_{1}\right)}-2 q_{1} q_{2}\left(\frac{1}{k_{1} q_{1}}+\frac{1}{k_{1} q_{2}}\right)\right. \\
& +\operatorname{Re}\left[\mathcal{A}_{\pi^{+} \pi^{-} \rightarrow \gamma \gamma}(s)\right]\left[3 k_{1} k_{2}+\frac{\left(k_{2} q_{1}\right)^{2}-\left(k_{1} k_{2}\right)\left(q_{1} q_{2}\right)}{k_{1} q_{1}}+\frac{\left(k_{1} q_{1}\right)^{2}-\left(k_{1} k_{2}\right)\left(q_{1} q_{2}\right)}{k_{2} q_{1}}\right] \\
& \left.+\frac{\left|\mathcal{A}_{\pi^{+} \pi^{-} \rightarrow \gamma \gamma}(s)\right|^{2}}{2}\left(k_{1} k_{2}\right)^{2}\right) .
\end{aligned}
$$

With the following definitions of the variables: $k_{1}=(\omega, \mathbf{k}), k_{2}=(\omega,-\mathbf{k}), q_{1}=(\omega, \mathbf{q})$, $q_{2}=(\omega,-\mathbf{q}), s=\left(q_{1}+q_{2}\right)^{2}=4 \omega^{2}$, the calculation of the cross section for this process gives

$$
\begin{aligned}
\sigma^{\pi^{+} \pi^{-} \rightarrow \gamma \gamma}(s) & =\frac{1}{16 \pi^{2}} \frac{1}{s \kappa} \int \frac{d^{3} k_{1}}{2 \omega_{1}} \frac{d^{3} k_{2}}{2 \omega_{2}} \delta^{4}\left(q_{1}+q_{2}-k_{1}-k_{2}\right)\left|T_{\pi^{+} \pi^{-} \rightarrow \gamma \gamma}(s)\right|^{2} \\
& =\sigma_{1}(s)+\sigma_{2}(s)+\sigma_{3}(s)
\end{aligned}
$$

with the partial cross sections

$$
\begin{aligned}
& \sigma_{1}(s)=16 \sigma_{0}\left(2-\kappa^{2}-\frac{1-\kappa^{4}}{2 \kappa} \ln \left[\frac{1+\kappa}{1-\kappa}\right]\right), \\
& \sigma_{2}(s)=4 \sigma_{0} s \operatorname{Re}\left[\mathcal{A}_{\pi^{+} \pi^{-} \rightarrow \gamma \gamma}(s)\right] \frac{1-\kappa^{2}}{\kappa} \ln \left[\frac{1+\kappa}{1-\kappa}\right], \\
& \sigma_{3}(s)=\sigma_{0} s^{2}\left|\mathcal{A}_{\pi^{+} \pi^{-} \rightarrow \gamma \gamma}(s)\right|^{2}
\end{aligned}
$$

corresponding to the Born, the interference and the quark substructure contributions, respectively, see Figs. 1 and 2. We have introduced the abbreviations $\sigma_{0}=\pi \alpha^{2} / 4 s \kappa$ and $\kappa^{2}=1-4 M_{\pi}^{2} / s$.

In order to give estimates if an enhancement of the cross sections causes an enhancement of the photon pair production rate, we calculate

$$
\frac{d N_{\gamma \gamma}}{d^{4} x d M}=4 M \int \frac{d^{3} p_{1}}{(2 \pi)^{3}} \int \frac{d^{3} p_{2}}{(2 \pi)^{3}} v_{\mathrm{rel}} \sigma^{\pi^{+} \pi^{-} \rightarrow \gamma \gamma} n_{\pi}\left(p_{1}\right) n_{\pi}\left(p_{2}\right) \delta\left(M^{2}-\left(p_{1}+p_{2}\right)^{2}\right)
$$

being the rate per space-time element with the relative velocity $v_{\text {rel }}\left(p_{1}, p_{2}\right)=\sqrt{1-M_{\pi}^{4} /\left(p_{1} p_{2}\right)^{2}}$ and the pion distribution function $n_{\pi}(p)=\left\{\exp \left(\sqrt{p^{2}+M_{\pi}^{2}} / T\right)-1\right\}^{-1}$. After integration over momenta and angles one obtains 


$$
\frac{d N_{\gamma \gamma}}{d^{4} x d M}=\frac{M^{2} T^{2}}{(2 \pi)^{4}} \sigma^{\pi^{+} \pi^{-} \rightarrow \gamma \gamma} \sqrt{M^{2}-4 M_{\pi}^{2}} \int_{\lambda}^{\infty} d x \frac{1}{e^{x}-1} \ln \left[\frac{1-\exp \left(F_{-}(x, T, \mu)\right)}{1-\exp \left(F_{+}(x, T, \mu)\right)}\right]
$$

where $\lambda=M_{\pi} / T$ and

$$
F_{ \pm}(x, T, \mu)=-x\left(\frac{M^{2}}{2 M_{\pi}^{2}(T, \mu)}-1\right) \pm \sqrt{x^{2}-\lambda^{2}} \sqrt{\left[\frac{M^{2}}{2 M_{\pi}^{2}(T, \mu)}-1\right]^{2}-1} .
$$

Similar expressions can be found in [18] for dilepton production rates. Note that the cross section $\sigma^{\pi^{+} \pi^{-} \rightarrow \gamma \gamma}=\sigma^{\pi^{+} \pi^{-} \rightarrow \gamma \gamma}\left(s=M^{2}, T, \mu\right)$ is medium dependent and obtained from (12).

In the following, we examine the change of the cross section for photon pair production and the photon production rate from two-pion annihilation in a hot and dense medium numerically. To this end, we use as an input the in-medium modifications of quark and meson properties which we have obtained from a chiral quark model at finite temperature and chemical potential.

\section{Numerical results}

In our model we fix the constituent quark mass in the vacuum at $m=300 \mathrm{MeV}$ and obtain at zero temperature and chemical potential the values for the meson masses and the pion decay constant given in Table 1. At finite temperature and chemical potential these values change due to medium effects what has been analyzed in several publications, e.g. $[14,15,19]$. In the following we study the behaviour of the cross sections at special points in the $T-\mu$ plane. The calculation of the quark and meson properties within the NJL model provides the values shown in Table 1 for $T=100 \mathrm{MeV}$ and $\mu=250 \mathrm{MeV}$ and $T=170 \mathrm{MeV}$ and $\mu=80$ $\mathrm{MeV}$. The choice of the latter set is motivated by a recent analysis of hadron spectra from heavy-ion collisions at SPS energies within a thermal model [20]. From the finite temperature studies of the chiral phase transition we know that due to the chiral symmetry restoration there occurs also an "inverse" Mott transition where the $\sigma$ meson mass drops below the twopion continuum threshold and thus the width for the $\sigma \rightarrow \pi \pi$ decay vanishes. Consequently the $\sigma$ pole contribution to cross section becomes enhanced close to the two-pion threshold. This effect has been paraphrased as "critical scattering" in the analysis of cross sections for hadronization [21] and two-photon production in a quark-meson plasma [11].

We have calculated the cross section for the annihilation of two charged pions into two photons and we have analyzed the temperature and density dependence of the different contributions (13-15) to the total cross section (12). Due to substructure effects we expect to

5 We have used a 3-dimensional cut off for regularization of the divergent integrals. 
obtain a strong enhancement of the total cross section in vicinity of the two-pion threshold. In Fig. 3 we show as our main result that the cross section for two-photon production is critically enhanced only in a narrow band of values in the $T-\mu$ plane which is closely related to the critical line for the chiral restoration transition.

Let us study this effect more in detail. The different partial contributions to the cross section as a function of the energy $s$ are given in Fig. 4. The upper panel shows the numerical results for the vacuum $(T=\mu=0)$ case where the main contribution comes from the Born diagram while the substructure has only a small contribution of about $10 \%$. The situation changes at finite temperature and chemical potential. While the Born term does not change, the substructure terms become important. As shown in the lower panel of Fig. 4, the $\sigma$ pole diagram gives the dominant contribution and the total cross section is strongly enhanced at threshold.

For the neutral pion annihilation we obtain qualitatively the same result. In this case the Born diagrams do not contribute at all and we have a drastic increase of the cross section at the threshold by three orders of magnitude according to the contribution of the $\sigma$ pole, Eq. (15). The numerical results are shown in Fig. 5.

In order to verify whether the strong enhancement of the cross section entails an enhancement in the production rate of photon pairs per space-time element we calculate this quantity according to Eq. (16) by folding the cross section with the pion distribution function Eq. (16) and the relative velocity. The numerical results are shown in Fig. 6. The corresponding

plot for the rates in the vacuum is not displayed, it is supressed by orders of magnitude. This plot clearly indicates the strong enhancement of the photon production rate in a narrow temperature band around the Mott transition temperature. In our numerical example we chose $\mu=80 \mathrm{MeV}$ where this effect happens at $T \approx 170 \mathrm{MeV}$, see Fig. 6 . For temperatures below and above this critical value the resonance is broad and not peaked. Hence, if we look to this process from the experimental point of view, the narrowing and enhancement of the $\sigma$ resonance along the transition line could be a hint to chiral symmetry restoration in heavy ion collisions.

\section{Conclusions}

In the present study we have investigated the medium dependence of the pion annihilation into a photon pair. Due to partial chiral symmetry restoration at finite temperatures and densities the $\sigma$ meson mass drops below the two pion threshold and appears as a bound state pole. This effect has several consequences. One of them is a strong enhancement of the cross section for pion annihilation in the two-photon channel as considered in the present work.

We have shown that the dramatic increase of the cross section for photon production appears in a narrow band in the $T-\mu$ plane which corresponds to the critical line of the chiral 
symmetry breaking/restoration transition. Thus the enhancement of photon pair production rate at threshold should belong to the set of observable effects for the suspected chiral symmetry restoration in ultra-relativisitc heavy ion collision experiments. However, the strong background due to $\pi^{0} \rightarrow 2 \gamma$ complicates the observation of this effect in these experiments.

In order to check the relevance of the present studies for experimental situations, a careful study of this process in a hydrodynamic scenario at the hadronisation transition is required. Such an analysis should include both the hadronic (pions) as well as quark effects under

the influence of a hydrodynamical evolution of the hot and dense system and is deferred to future work.

\section{Acknowledgement}

M.K.V. is grateful to J. Hüfner for fruitful discussions. One of us (M.K.V.) acknowledges financial support provided by INTAS under Grant No. W 94-2915 and by the Max-PlanckGesellschaft as well as the hospitality of the AG "Theoretische Vielteilchenphysik" at the University of Rostock where part of this work has been done. E.A. Kuraev acknowledges financial support provided by INTAS under Grant No. W 93-0239. S.S. is grateful for the financial support provided by the MINERVA foundation.

\section{References}

[1] QUARK MATTER '96, P. Braun-Munzinger, H.J. Specht, R. Stock and H. Stöcker, Nucl. Phys. A 610 (1996).

[2] G. Chanfray, R. Rapp and J. Wambach, Phys. Rev. Lett. 76 (1996) 368.

[3] G.Q. Li, C.M.Ko and G.E. Brown, Phys. Rev. Lett. 75 (1995) 4007.

[4] H.-J. Schulze and D. Blaschke, Phys. Lett. B 386 (1996) 429.

[5] Review of Particle Properties, Phys. Rev. D 54 (1996) 1.

[6] M. Svec, A. de Lesqnen and L. van Rossum, Phys. Rev. D 46 (1992) 949;

S. Ishida et al., Prog. Theor. Phys. 95 (1996) 745.

[7] M.K. Volkov, Sov. J. Part. and Nucl. 17 (1986) 186.

[8] M.K. Volkov and A.A. Osipov, Sov. J. Nucl. Phys. 41 (1985) 659.

[9] T. Hatsuda and T. Kunihiro, Phys. Lett. B 185 (1987) 304.

[10] M.K. Volkov, E.A. Kuraev, D. Blaschke, G. Röpke and S. Schmidt, Dubna Preprint E2-96-470 (1996). 
[11] P. Rehberg, Yu.L. Kalinovsky and D. Blaschke, Nucl. Phys. A 622 (1997) 478.

[12] H.A. Weldon, Phys. Lett. B 274 (1992) 133.

[13] A.E. Dorokhov, J. Hüfner, S. Klevansky and M.K. Volkov, Chinese J. Phys. 34 (1996) 901;

A.E. Dorokhov et al., Z. Phys. C 75 (1997) 127.

[14] S. Schmidt, D. Blaschke and Yu.L. Kalinovsky, Phys. Rev. C 50 (1994) 435; Z. Phys. C 66 (1995) 85.

[15] S. Schmidt, Ph.D. thesis, Rostock University, 1995 (unpublished);

D. Blaschke, Yu.L. Kalinovsky, G. Röpke, S. Schmidt and M.K. Volkov, Phys. Rev. C 53 (1996) 2394.

[16] Y. Nambu and G. Jona-Lasinio, Phys. Rev. 122 (1961) 345;

M.K. Volkov, Ann. Phys. (NY) 159 (1984) 282;

U. Vogl and W. Weise, Progr. Part. Nucl. Phys. 27 (1991) 195;

S.P. Klevansky, Rev. Mod. Phys. 64 (1992) 649;

T. Hatsuda and T. Kunihiro, Phys. Rep. 247 (1994) 221.

[17] M.K. Volkov, Theor. Math. Phys. 101 (1994) 1473.

[18] F. Karsch, K. Redlich and L. Turko, Z. Phys. C 60 (1993) 519, and references therein.

[19] D. Ebert, Yu.L. Kalinovsky, L. Münchow and M.K. Volkov, Int. J. Mod. Phys. A 8 (1993) 1295 .

[20] P. Braun-Munzinger, J. Stachel, J.P. Wessels and N. Xu, Phys. Lett. B 365 (1996) 1.

[21] J. Dolejší, W. Florkowski and J. Hüfner, Phys. Lett. B 349 (1995) 18. 


\begin{tabular}{|c|c|c|c|c|c|c|}
\hline Set \# & $T[\mathrm{MeV}]$ & $\mu[\mathrm{MeV}]$ & $m[\mathrm{MeV}]$ & $M_{\pi}[\mathrm{MeV}]$ & $M_{\sigma}[\mathrm{MeV}]$ & $f_{\pi}[\mathrm{MeV}]$ \\
\hline 1 & 0 & 0 & 300 & 140 & 610 & 93 \\
\hline 2 & 100 & 252 & 154 & 165 & 338 & 55 \\
\hline 3 & 170 & 80 & 150 & 161 & 330 & 55 \\
\hline
\end{tabular}

Table 1

Typical examples for sets of parameter values obtained within the chiral quark model of Refs. $[14,15]$. Set \#1 corresponds to the vacuum case, the sets \#2 and \#3 correspond to points in the $T-\mu$ plane which are relevant for a discussion of the hadronization transition in heavy-ion collisions. 

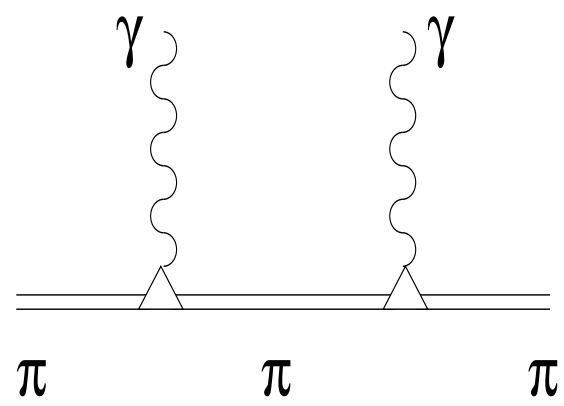

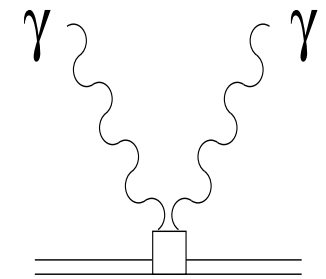

$\pi$ $\pi$

Fig. 1. Feynman diagrams corresponding to the Lagrangian (1).

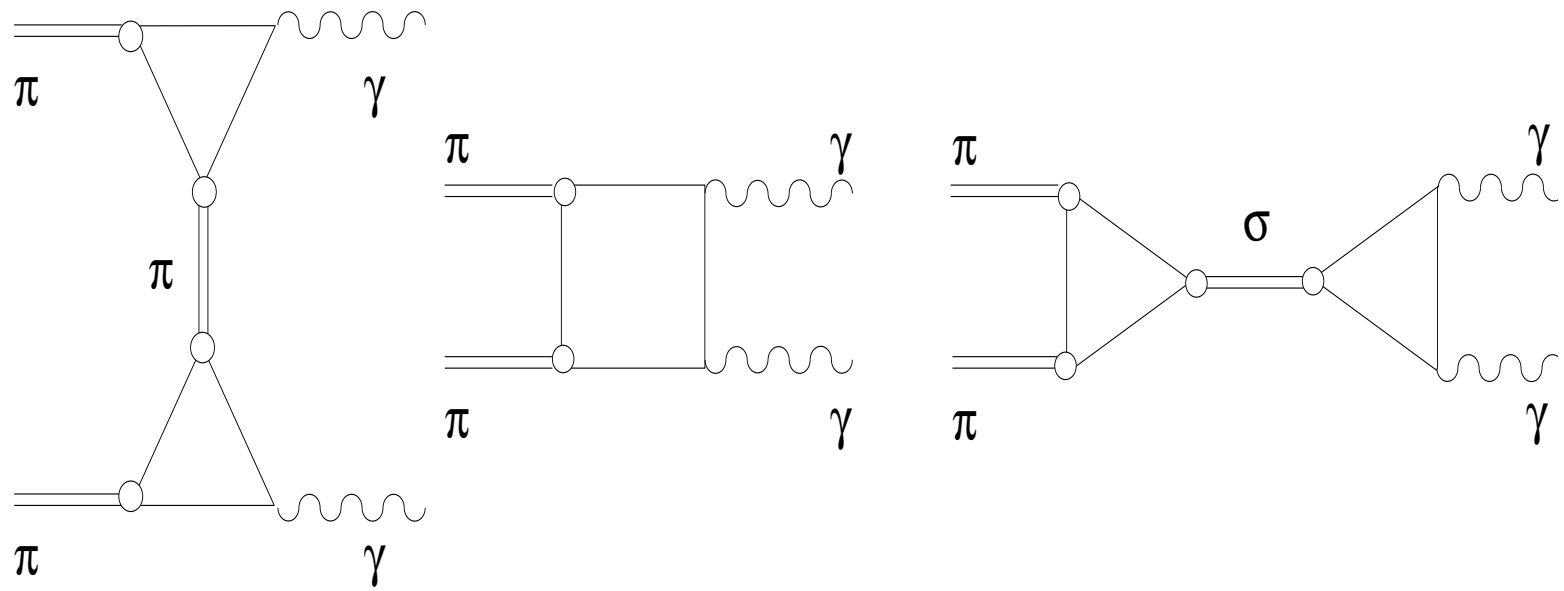

(a)

(b)

(c)

Fig. 2. Feynman diagrams contributing to the process $\pi \pi \rightarrow \gamma \gamma$ including quark substructure effects in lowest order $\left(1 / N_{c}\right)$ (refLbox)-(4). The lowest order gradient expansion of this set of diagrams corresponds to that of Fig. 1. 


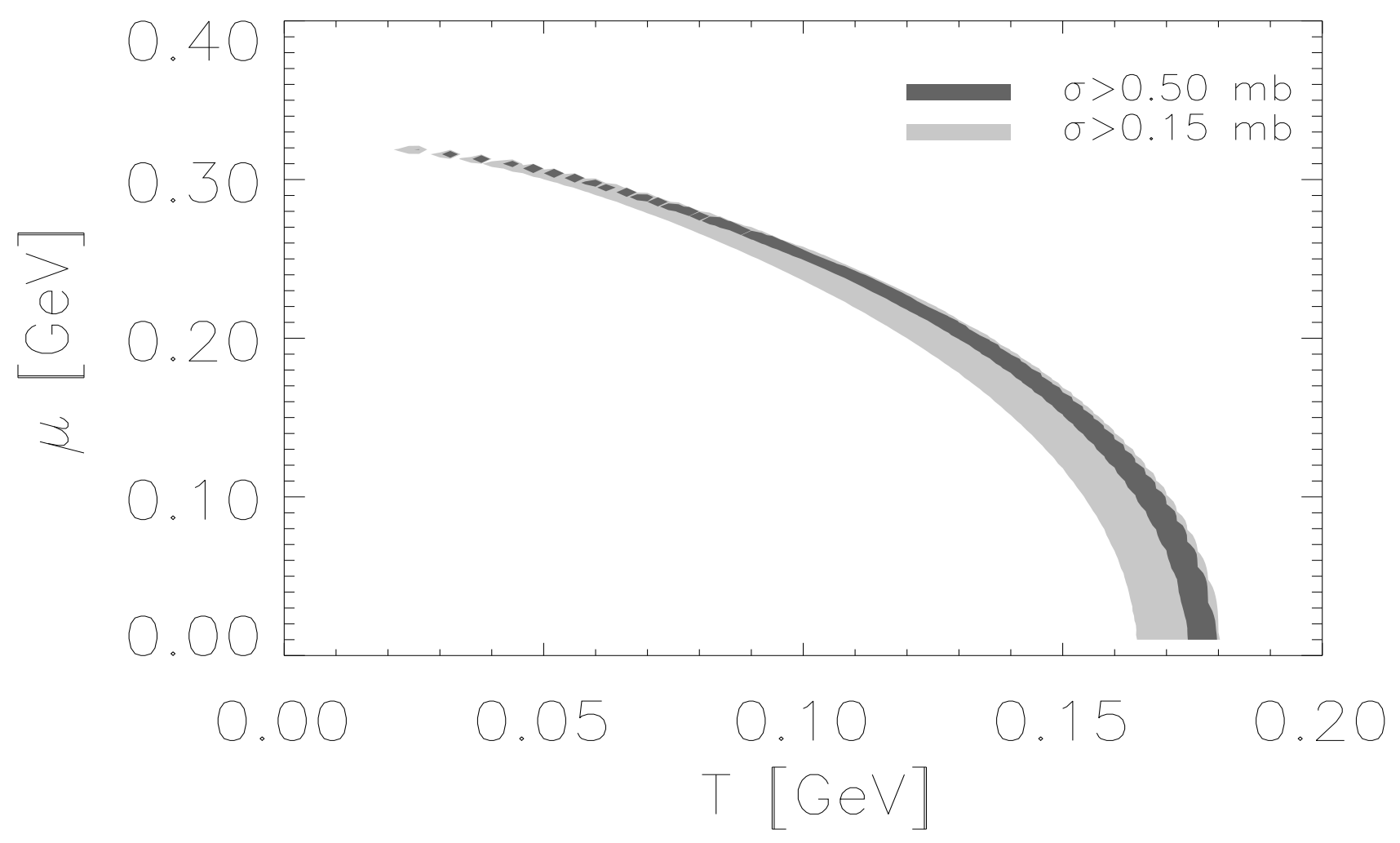

Fig. 3. Enhancement of the total cross section for $\pi \pi \rightarrow \gamma \gamma$ in the $T-\mu$ plane due to chiral symmetry restoration shown for $\sqrt{s-4 M_{\pi}^{2}}=10 \mathrm{MeV}$. 


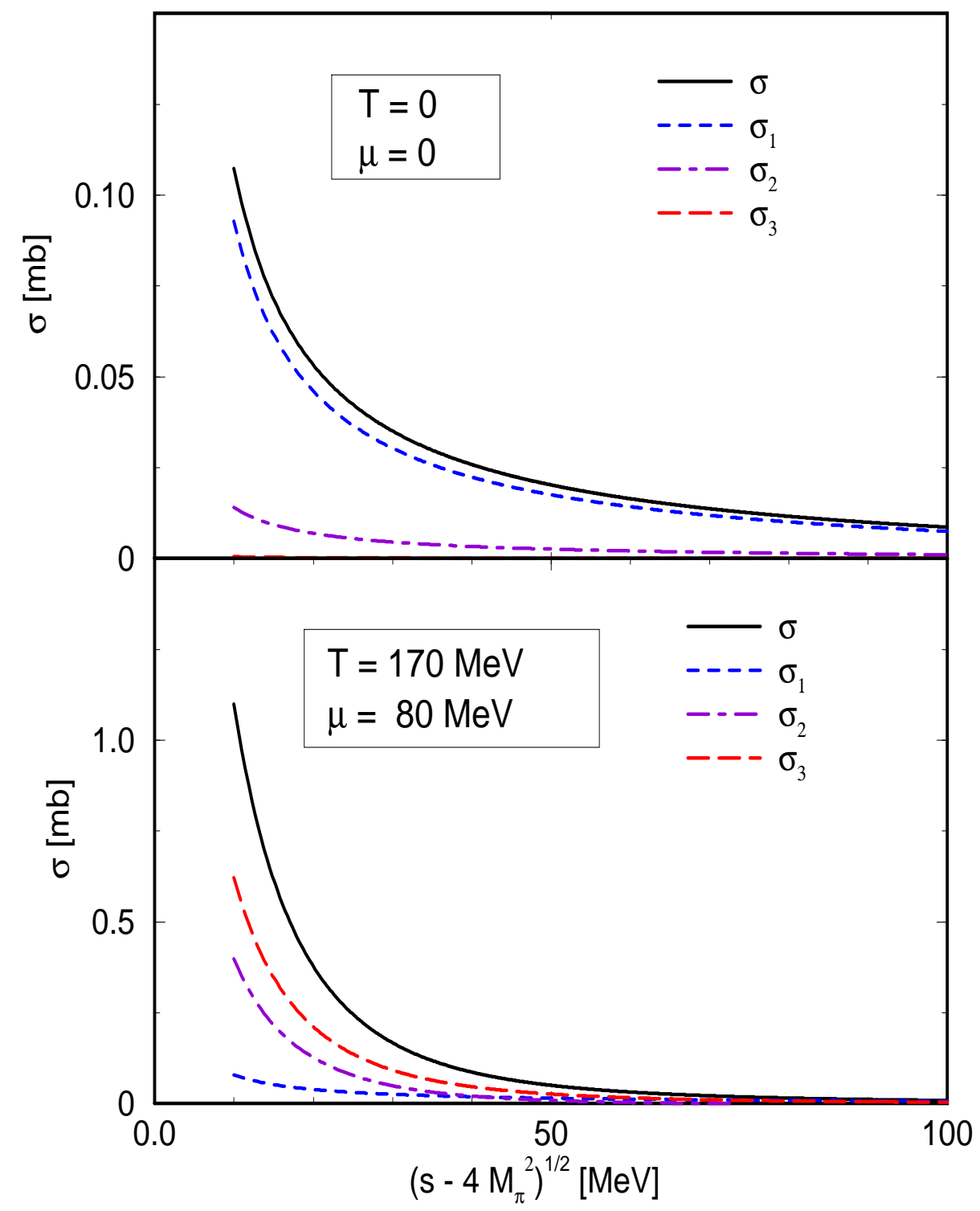

Fig. 4. Cross section for the annihilation of charged pions into photon pairs in the vacuum $T, \mu=0$ (upper panel) and in a hot, dense medium at $T=170 \mathrm{MeV}, \mu=80 \mathrm{MeV}$ (lower panel). This point in the $T-\mu$ plane has been chosen in accordance with recent analyses of hadron spectra at CERN-SPS [20]. The partial cross section for the Born process $\left(\sigma_{1}\right)$ is dominant in the vacuum, the quark substructure contributions contained in $\sigma_{3}$ become dominant at finite $T, \mu$ in the vicinity of the chiral restoration transition because of the strong enhancement of the $\sigma$ pole contribution. The interference contribution is denoted by $\sigma_{2}$. 


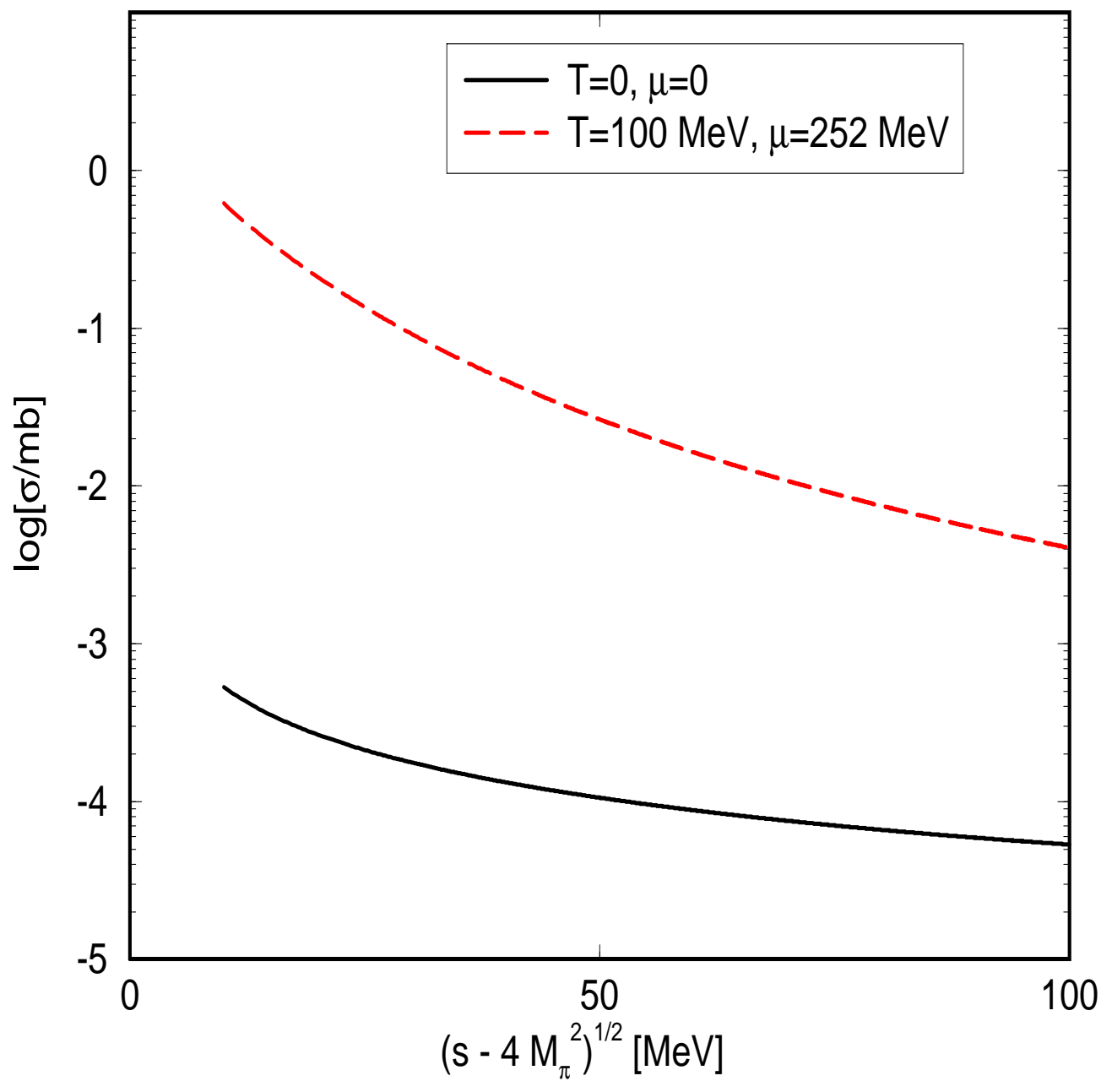

Fig. 5. Cross section for the annihilation of neutral pions into photon pairs in the vacuum $T, \mu=0$ and in a hot, dense medium at $T=100 \mathrm{MeV}, \mu=250 \mathrm{MeV}$. 


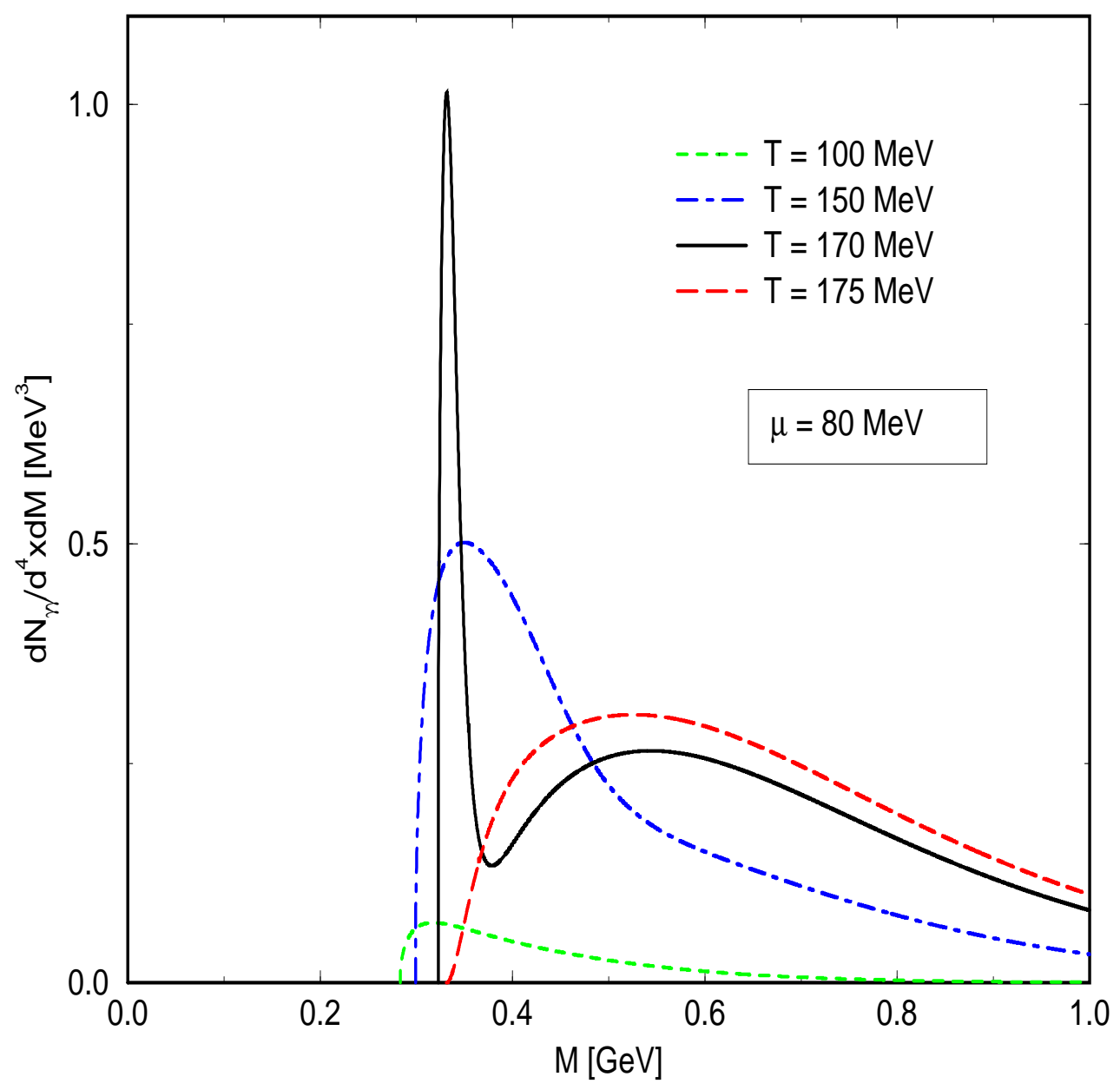

Fig. 6. Production rate for photon pairs with invariant mass $M$ by annihilation of charged pions for fixed chemical potential and different temperatures below and above the critical line, compare Fig. 3. 